\title{
Estructura Factorial e Invarianza Métrica de la Versión Chilena de la Heartland Forgiveness Scale (HFS)
}

\author{
Factorial Structure and Metric Invariance of the Chilean Version of the Heartland \\ Forgiveness Scale (HFS)
}

\author{
Mónica Guzmán-González ${ }^{1}$, Carlos Calderón², Katherine Alfaro Escobedo ${ }^{3}$, \\ Karin Phunhon Marangunic ${ }^{4}$ y Karina Toro Aguirre ${ }^{5}$
}

\begin{abstract}
Resumen
El estudio tuvo como objetivo evaluar la estructura factorial e invarianza métrica de la versión chilena del Heartland Forgiveness Scale, que mide el perdón disposicional a sí mismo, a otros y a las situaciones. Para ello, se evaluó una muestra de 549 adultos chilenos (50.6\% mujeres, 49.4\% hombres) con edades entre los 18 y 65 años $(M=40.65$ años, $\mathrm{DE}=13.83)$. Los resultados evidenciaron que una estructura de seis factores es la que obtuvo el mejor ajuste dentro de las diferentes soluciones examinadas. Esta configuración considera factores referidos a los tres objetos del perdón, subdivididos a su vez en dimensiones positivas y negativas. La estructura factorial retenida fue invariante entre hombres y mujeres. Además, se identificaron asociaciones significativas con una medida de perdón episódico, así como con indicadores de salud mental y satisfacción con la vida en las direcciones esperadas. Se discuten las implicancias de estos hallazgos.
\end{abstract}

Palavras-chave: perdón disposicional, estructura factorial, dimensión positiva y negativa del perdón, invarianza métrica

\begin{abstract}
The aim of the study was to evaluate the factor structure and metric invariance of the Chilean version of the Heartland Forgiveness Scale, which measures dispositional forgiveness to self, others and situations. For this purpose, a sample of 549 Chilean adults (50.6\% women, $49.4 \%$ men) aged between 18 and 65 years $(\mathrm{M}=40.65$ years, $\mathrm{SD}=13.83)$ was evaluated. The results showed that a six-factor structure is the one that obtained the best fit among the different solutions examined. This configuration considers factors referring to the three objects of forgiveness, which are further divided into positive and negative dimensions. The retained factor structure was invariant between men and women. In addition, significant associations were identified with a measure of episodic forgiveness, as well as with indicators of mental health and life satisfaction in the expected directions. Implications of these findings are discussed.
\end{abstract}

Keywords: dispositional forgiveness, factorial structure, positive and negative dimensions of forgiveness, metric invariance

\footnotetext{
${ }^{1}$ Doctora en Psicología. Escuela de Psicología, Universidad Católica del Norte, Chile. Profesora Titular. Av. Angamos 0610, Antofagasta, Región de Antofagasta, Chile. Tel.: +56 55 2355822. Correo: moguzman@ucn.cl (Autor de correspondencia)

${ }^{2}$ Doctor en Metodología de las Ciencias del Comportamiento y de la Salud. Universidad Católica del Norte, Chile. Profesor Asistente. Av. Angamos 0610, Antofagasta, Región de Antofagasta, Chile. Tel.: +56 55 2355822. Correo: ccalderon@ucn.cl

${ }^{3}$ Magister en Psicología Clínica. Observatorio de Infancias y Juventudes. Líder de Intervención. Universidad Católica del Norte, Chile. Av. Angamos 0610, Antofagasta, Región de Antofagasta, Chile. Correo: katherine.alfaro@ce.ucn.cl

${ }^{4}$ Magister en Psicología Cognitiva Social. Escuela de Psicología, Universidad Católica del Norte, Chile. Av. Angamos 0610, Antofagasta, Región de Antofagasta, Chile. Tel.: +56 55 2355822. Correo: karinphunhon@gmail.com

${ }^{5}$ Magister en Psicología Clínica. Escuela de Psicología, Universidad Católica del Norte, Chile. Supervisora Prácticas Clínicas. Av. Angamos 0610, Antofagasta, Región de Antofagasta, Chile. Tel.: +56 55 2355822. Correo: ktoroaguirre@ gmail.com
}

Revista Iberoamericana de Diagnóstico y Evaluación - e Avaliação Psicológica. RIDEP · No61 · Vol.4 · 117-130 · 2021

ISSN: 1135-3848 print /2183-6051online 


\section{Introducción}

El estudio del perdón ha concitado un interés sostenido dentro de la comunidad científica dada la evidencia que existe respecto de su asociación con medidas de bienestar (Abu-Raiya \& Ayten, 2020; Akhtar \& Barlow, 2018; Kaleta \& Mróz, 2018a), con indicadores de salud tanto física como mental (e.g., Fincham, 2015; Gall \& Billodeau, 2020; Griffin et al., 2015; Jung et al., 2019; Kaleta \& Mróz, 2020; Lee \& Enright, 2019; McCullough \& Witvliet, 2002; Lee \& Enright, 2019; Tuck \& Anderson, 2014), al igual que con relaciones interpersonales más satisfactorias y un mejor manejo de conflictos (Aalgaard et al., 2016; Fincham et al., 2004; Sheldon et al., 2014; Wilkowski et al., 2010). Asimismo, el perdón se ha posicionado como un objetivo terapéutico en personas en distintas condiciones vitales, con efectos positivos sobre síntomas como la depresión, la ansiedad y el estrés (Akhtar \& Barlow, 2018; Wade, Hoyt et al., 2014).

En la literatura sobre este tópico, se identifican diversas definiciones del perdón, sin embargo, un elemento en el cual distintas conceptualizaciones convergen, es que este puede ser concebido como una estrategia de afrontamiento ante la vivencia de una herida interpersonal, que implica la disminución del resentimiento y las respuestas negativas asociadas, junto con el incremento de sentimientos más neutros o incluso de benevolencia (Enright \& Fitzgibbons, 2015; Strelan \& Covic, 2006; Worthington \& Sandage, 2016). Dependiendo de la fuente de la herida, el perdón puede tener como objeto a otros (perdón interpersonal), a sí mismo, o a situaciones que escapan al propio control (Thompson et al., 2005). El perdón interpersonal alude al conjunto de cambios motivacionales, a través de los cuales la persona reemplaza respuestas destructivas, por ejemplo, la hostilidad y el deseo de tomar revancha o distanciarse de quien cometió la ofensa, por respuestas más neutras o constructivas y benevolentes (McCullough et al., 2001; Thompson et al. 2005). El perdón a sí mismo por su parte, es entendido como el proceso mediante el cual la persona abandona la auto recriminación y ante el reconocimiento genuino que ha cometido un error, adopta una mirada más benevolente y compasiva de sí misma (Enright, 1996; Hall \& Fincham, 2005). El perdón a las situaciones alude a un concepto más global que ocurre ante situaciones percibidas como fuera del propio control, como es el caso de una enfermedad, o un desastre natural e implica la transformación de respuestas negativas como la rabia y tristeza hacia otras más neutras o incluso positivas (Thompson et al., 2005). Si bien el perdón a situaciones ha sido objeto de cuestionamiento por parte de algunos autores bajo el argumento que el perdón se dirige a personas (e.g., Enright, 1989), Thompson et al. señalan que esta diferenciación es relevante para capturar el perdón en contextos donde la fuente de la ofensa es menos clara, por ejemplo una enfermedad (Thompson et al., 2005).

Por otra parte, dentro de la literatura del perdón, una discusión que se ha instalado tiene que ver con la dimensionalidad de este constructo (Guzmán, 2010; Rosales et al., 2018). Algunos autores sostienen que este tendría un carácter unidimensional, es decir, que involucra un descenso en la negatividad de las emociones, pensamientos y conductas hacia la fuente de la ofensa y que no requiere, necesariamente, una orientación positiva hacia el otro (Gordon \& Baucom, 1998; Gordon, 2003). De hecho, Thompson et al. (2005) sostienen que el cambio en la valencia de las respuestas de negativas a neutras sería condición necesaria y suficiente para cumplir los criterios del perdón.

Sin embargo, hay otros autores que sostienen que el perdón estaría compuesto de dos facetas: la dimensión negativa y positiva del perdón (Fincham, 2015; Kaleta \& Mróz, 2016; Rye et al., 2001; Worthington \& Wade, 1999). La primera hace alusión a la reducción de pensamientos, emociones y conductas negativas hacia el objeto de la transgresión (también descrita como la disminución del unforgiveness); esta dimensión alude por tanto a un descenso en respuestas como la rumiación, la rabia y el deseo de venganza; o bien la culpa y la auto recriminación cuando el objeto del perdón es uno mismo (Fincham \& Beach, 2002; Gordon et al., 2009; McCullough et al., 2009). Por otro lado, la dimensión positiva refiere al desarrollo de pensamientos, sentimientos y conductas más benevolentes, y mayor compasión, que supone un cambio en la percepción de otros, de sí mismo o del mundo 
(Fincham, 2015; McCullough \& Hoyt, 2002; Wade \& Worthington, 2003). En apoyo a esta segunda posición, se ha evidenciado que ambas dimensiones del perdón tienen diferentes correlatos y consecuencias (Fincham \& Beach, 2002). A modo de ejemplo, se ha identificado que solo la dimensión negativa del perdón se asocia a la violencia psicológica y que la dimensión positiva y no la negativa se vincula a patrones de comunicación más constructivos (Fincham \& Beach, 2002). En estudios más recientes se concluyó que solo la dimensión negativa del perdón se asocia al neuroticismo (Kaleta \& Mróz, 2018a), y que la evitación y venganza se asocian a la edad, pero no la dimensión de benevolencia (Ghaemmaghami et al., 2011; Kaleta \& Mróz, 2018b).

Adicionalmente, en la comprensión del perdón, se han distinguido tres niveles de especificidad: perdón episódico o estado, diádico y disposicional. El primero hace alusión al perdón de una situación o herida en particular; el perdón diádico es entendido como la capacidad de perdonar dentro de una relación específica (Kachadourian et al., 2004; Thompson et al., 2005). Finalmente, el perdón disposicional es entendido como una característica estable o rasgo de personalidad que alude a la tendencia a perdonar a través del tiempo y las situaciones (Thompson et al., 2005), siendo este el foco del presente estudio.

Para la medición del perdón disposicional se encuentran disponibles una serie de instrumentos, tales como el Transgression Narrative Test of Forgiveness (TNTF; Berry et al., 2001), la Tendency to Forgive Scale (TTF; Brown, 2003) y la Forgiveness using Implicit Association Test (FIAT; Bueschel, 2010), por mencionar algunos. Sin embargo, la medida más ampliamente utilizada es la Heartland Forgiveness Scale (HFS), desarrollada por Thompson et al., (2005). La HFS es un instrumento de carácter multidimensional que tiene la ventaja de incorporar los tres objetos del perdón (a otros, a sí mismo y a las situaciones), a diferencia de las otras escalas mencionadas, orientadas únicamente al perdón interpersonal. Por tanto, su carácter más versátil lo hacen útil para su uso en distintos ámbitos. La HFS ha sido validada para su uso en distintos contextos, disponiéndose de la versión japonesa (Osanai \& Furukawa, 2005), india (Dahiya \& Rangnekar, 2018), turca (Bugay et al., 2012), polaca (Kaleta et al., 2016), portuguesa (Ikedo et al., 2020), española (Gallo-Giunzioni et al., 2021) e italiana (Consoli et al., 2020). Originalmente, los autores presentaron a la HFS como una escala compuesta por tres dimensiones de seis ítems cada una, que aluden a los tres objetos del perdón (Thompson \& Snyder, 2003). Sin embargo, en un estudio posterior proponen un modelo alternativo más complejo (Thompson et al., 2005). De acuerdo a esta propuesta, la escala estaría compuesta por seis factores de primer orden, correspondientes a factores de valencia positiva y negativa de los tres objetos del perdón (otros, sí mismo y situaciones). Adicionalmente, incluye dos tipos de factores de segundo orden: tres factores sustantivos que acumulan la varianza producto de los tres objetos de perdón, y dos factores de valencia afectiva, que acumulan la varianza producto de la dirección de los ítems. Ambos tipos de factores, valencia afectiva $y$ objetos de perdón se presentan como no correlacionados. Por tanto, el modelo correspondería a un modelo mixto bifactor de segundo orden.

Pese al amplio uso de la HFS en la investigación, los estudios que han examinado la estructura factorial del HFS no siempre han replicado el modelo jerárquico propuesto por Thompson et al. (2005). Si bien la versión italiana logró replicar la estructura compleja propuesta por los autores (Consoli et al., 2020), existe también evidencia que no es consistente con estos hallazgos. Por ejemplo, la versión japonesa (Osanai \& Furukawa, 2005), propone una estructura de dos factores, un factor que agrupa los ítems de las dimensiones de perdón a sí mismo y a las situaciones, y un segundo factor de perdón a otros. Por otro lado, la versión polaca (Kaleta et al., 2016), propone una estructura de tercer orden, con seis factores de primer orden en el que se distinguen las dimensiones negativa y positiva del perdón con sus tres objetos, dos de segundo (valencia positiva y valencia negativa), y un factor general de perdón. En un estudio reciente, llevado a cabo en una muestra española, los autores proponen una versión similar a la de Thompson et al. (2005), aunque eliminando 10 de los 18 ítems originales (Gallo-Giunzioni et al., 2021). En esta 
propuesta fueron excluidos los ítems de valencia negativa y el factor de perdón a las situaciones quedó conformado sólo por dos ítems, lo cual podría debilitar la validez de contenido de la escala (Abad, Olea, Ponsoda, \& García, 2014; Martínez Arias, Hernández Lloreda, \& Hernández Lloreda, 2013; Sireci, 1998; Suen, 1990), al hacer una depuración llevada a cabo por criterios solo estadísticos, sin considerar aspectos de representatividad y relevancia (Sireci, 2003). Adicionalmente, modelos con tres o menos indicadores por factor latente presenta problemas de identificación (Brown, 2006).

Debido a la inconsistencia presente en la literatura previa, es que se hace necesario disponer de mayor evidencia empírica que contribuya al debate respecto a la estructura interna de la HFS, especialmente cuando se usa en contextos distintos. Adicionalmente, se observa que en los estudios de validación de la HFS disponibles, no se ha explorado la equivalencia/invarianza de medida según sexo, cuestión relevante toda vez que las comparaciones entre hombres y mujeres suele ser una práctica frecuente en la investigación sobre el perdón (Miller \& Worthginton, 2010; Miller et al., 2008; Rey \& Extremera, 2016).

Por otro lado, en el caso particular de Chile, si bien se cuenta con medidas validadas del perdón, tales como la Transgression Related Interpersonal Motivations (TRIM-18; Guzmán-González et al., 2014) y el Cuestionario de Perdón a la ex pareja (CPDS; Guzmán-González et al., 2019), ambas se encuentran orientadas al perdón interpersonal. Hasta la fecha, no hay instrumentos, adaptados al contexto chileno, que evalúen el perdón como característica estable y con distintos objetos.

Por tanto y a la luz de los antecedentes expuestos, el objetivo de este estudio fue examinar la estructura factorial de la versión chilena de la HFS. Secundariamente, y como forma de aportar evidencia adicional relativa a la estructura de este instrumento, se evaluó la invarianza factorial de la escala según sexo y se examinó la asociación de las dimensiones de la HFS con una medida de perdón episódico, así como con indicadores de salud mental y bienestar (satisfacción con la vida). Dada la posibilidad que los factores de valencia afectiva no correspondan a factores instrumentales o de método (dirección de los ítems), sino más bien a dimensiones teóricamente distinguibles (dimensión negativa y positiva del perdón) (Fincham, 2015), es que hipotetizamos la existencia de un modelo de seis factores que distingue ambas dimensiones, para cada objeto del perdón. En segundo lugar, propusimos que la HFS será invariante según sexo, y, por último, que se observarán asociaciones diferenciales entre los puntajes de las subescalas de la HFS y una medida de perdón episódico, así como con indicadores de salud mental y satisfacción con la vida.

\section{Método}

\section{Participantes}

La muestra fue de carácter no probabilístico, estratificada por sexo, y estuvo compuesta de manera inicial por 556 participantes de las zonas norte, centro y sur de Chile. Los criterios de inclusión fueron tener entre 18 y 65 años de edad y poseer nacionalidad chilena. Tras el examen preliminar de los datos, se excluyeron 3 casos por un elevado número de datos ausentes y 4 casos por ser identificados como atípicos. Por lo tanto, la muestra final quedó compuesta por 549 personas, distribuidas en 278 mujeres $(50.6 \%)$ y 271 hombres (49.4\%). El promedio de edad de las mujeres fue de 39.87 años $(\mathrm{DE}=13.24)$ y el promedio de edad de los hombres fue de 40.24 años $(\mathrm{DE}=13.55)$. Respecto del estado civil, un $52.3 \%$ de los participantes declaró ser soltera/o, mientras que un $23.6 \%$ ser casada/o, un $22.3 \%$ señaló encontrarse separada/o o divorciada/o y el $1.8 \%$ ser viuda/o. En cuanto a la distribución según nivel educacional, un $32.9 \%$ declaró tener estudios superiores, un $58.1 \%$ estudios secundarios, mientras que un $9 \%$ indicó poseer educación media incompleta o menor.

\section{Instrumentos}

Junto con el registro de las características sociodemográficas correspondientes a sexo, edad, estado civil y nivel educacional, se aplicaron los siguientes instrumentos:

Heartland Forgiveness Scale (HFS; Thompson et al., 2005). Esta escala, orientada a medir el perdón disposicional, está compuesta por 18 ítems, distribuidos en tres subescalas de 6 reactivos cada una: el perdón a sí mismo ("Me cuesta mucho aceptarme a mí mismo/a cuando me 
he equivocado"), a otros ("Sigo siendo duro/a con quienes me han lastimado") y a situaciones que escapan del propio control y/o del control de un tercero ("Es muy difícil para mí aceptar situaciones negativas que no son culpa de nadie"). Cada dimensión posee tres ítems de valencia positiva y tres de valencia negativa. Los reactivos están en formato Likert, en una escala de 1 (muy falso para mí) a 7 (muy verdadero para mí). Puntajes más altos indican mayor disposición a perdonar. La HFS ha demostrado propiedades psicométricas adecuadas, incluyendo índices de consistencia interna apropiados, con valores Alfa de Cronbach fluctuantes entre .84 y .87 para la puntuación total de la escala, y entre .71 a .83 para las subescalas (Thompson \& Snyder, 2003).

\section{Transgression Related Interpersonal} Motivations Inventory (TRIM-18; McCullough et al., 2006), en su versión validada por Guzmán et al. (2014), es un instrumento de auto-reporte que tiene por objetivo evaluar el perdón episódico. Está conformado por 18 preguntas divididas en tres subescalas: la motivación a la evitación de quien cometió la ofensa, conformada por 7 ítems ("Mantengo entre nosotros la mayor distancia posible"), la motivación a buscar venganza ("Quiero verlo sufrir y en estado de miseria"), con 5 ítems, y 6 ítems que componen la dimensión de benevolencia ("He dejado atrás el dolor y el resentimiento"). Presenta un formato de respuesta de tipo Likert con una escala de 5 puntos, siendo 1 totalmente en desacuerdo y 5 totalmente de acuerdo, por lo que un puntaje elevado devela una mayor tendencia a la evitación, venganza y benevolencia respectivamente. El TRIM-18 presenta índices adecuados de fiabilidad y validez para su uso en el contexto chileno, evidenciando consistencia interna adecuada y buen ajuste a los datos en una estructura factorial tridimensional (Guzmán et al., 2014). Para este estudio, los índices de consistencia interna fueron $.86, .81 \mathrm{y}$ .79 para la escala de evitación, venganza y benevolencia respectivamente.

Versión chilena abreviada de las Escalas de Depresión, Ansiedad y Estrés (DASS - 21). Esta fue traducida y adaptada en Chile por Vinet et al. (2012). El DASS-21 (Antony et al., 1998) es un instrumento de autoinforme diseñado para medir la presencia de sintomatología depresiva, de ansiedad y estrés en la última semana. Está constituido por 21 ítems en una escala Likert de 4 categorías, con una puntuación entre 0 y 3 puntos, siendo 0 "no describe nada de lo que me pasó o sentí en la semana" y 3 sí, esto me pasó mucho, o casi siempre", distribuidos en tres subescalas con 7 ítems cada una: depresión ("No podía sentir nada positivo"), ansiedad ("Me di cuenta que tenía la boca seca") y estrés ("Sentí que estaba muy nervioso"). Un puntaje elevado devela mayor presencia de síntomas depresivos, de ansiedad y estrés respectivamente. Para este estudio, la confiabilidad de las escalas de depresión, ansiedad y estrés fue de $.88, .85$ y .89 respectivamente.

Satisfacción con la Vida. Se evaluó con la Escala de Satisfacción con la Vida (SWLS) de Diener et al. (1985), adaptada al contexto chileno por Vera-Villarroel et al., (2012). Este cuestionario de auto-reporte mide el juicio global que hacen las personas sobre la satisfacción con su propia vida. Se compone de 5 ítems en formato Likert con una escala de 7 puntos, siendo 1 "totalmente en desacuerdo" y 7 "totalmente de acuerdo" (ejemplo: "Las condiciones de mi vida son excelentes"). Puntuaciones más altas un indican mayor nivel de satisfacción con la vida. La SWLS presenta adecuados indicadores de consistencia interna, con un Alfa de Cronbach que oscila de .79 a .89, además de índices apropiados de fiabilidad y validez para su uso en el contexto chileno. Para este estudio, la confiabilidad de este instrumento fue de .79 .

\section{Procedimiento}

El estudio contó con la aprobación del Comité de Ética de la Universidad Católica del Norte y constó de dos fases. Una primera etapa correspondió a la realización de la adaptación lingüística del instrumento para su uso en el contexto chileno siguiendo las directrices de la Comisión Internacional de Test (ITC) para la adaptación cultural de test (Muñiz \& Hambleton, 1996; Muñiz, Elosúa, \& Hambleton, 2013). En dicho proceso se llevó a cabo una traducción del idioma original al español, una traducción inversa y finalmente, un comité de cuatro jueces expertos evaluó ambas y resolvió discrepancias. Posteriormente, se realizó un pilotaje de la escala aplicando el instrumento a una muestra de 162 personas, para luego realizar las modificaciones que se consideraron necesarias, referidas a 
cambios lingüísticos menores a 2 ítems, que mantuvieron el significado de la versión original.

La etapa 2 correspondió a la aplicación del cuestionario en su versión final. Esta aplicación se llevó a cabo en distintas ciudades de Chile, pertenecientes a la zona norte, centro y sur del país. El acceso a la muestra se realizó ocupando diferentes estrategias: visitas a lugares públicos, redes sociales, contacto con instituciones públicas y educacionales a fin de coordinar la toma de datos con funcionarios de los establecimientos. $\mathrm{Al}$ momento de la aplicación, se entregó información sobre los objetivos del estudio y sobre el carácter anónimo, confidencial y voluntario de la participación en el mismo. Seguidamente, se procedió a la firma del consentimiento informado y luego, cada participante respondió de manera individual la encuesta en formato impreso. La confidencialidad de los datos fue resguardada en función de la Ley Chilena 19.628 de Protección de la vida privada.

\section{Estrategia de análisis de datos}

Antes de proceder al análisis de datos, se examinó la matriz de datos, con el objeto de detectar y manejar datos ausentes y casos atípicos. Para el examen de la estructura factorial se llevó a cabo un Análisis Factorial Confirmatorio con el método Máxima Verosimilitud con media y varianza corregida (MLMV) disponible en Mplus (Muthén \& Muthén, 1998-2015) a fin de evitar el efecto de la no normalidad de los datos sobre los estimadores. Se compararon tres modelos alternativos: el modelo original (modelo A) propuesto por los autores de la HFS (Thomson \& Snyder, 2003), compuesto por 3 factores de primer orden correspondientes a las dimensiones de perdón a sí mismo, hacia otros y a situaciones; un modelo de seis factores de primer orden, el cual considera factores referidos a los tres objetos de perdón, pero divididos en valencia positiva y valencia negativa y en el que cada factor está compuesto de tres ítems (modelo B); y por último, el modelo mixto bifactor de segundo orden propuesto posteriormente por Thompson et al., 2005 (modelo C). Este último modelo especifica seis factores de primer orden para los ítems de valencia positiva y negativa de cada una de las dimensiones del perdón disposicional (sí mismo, otros, situaciones), a fin de manejar la varianza sistemática relacionada con la dirección de los ítems. Dichos factores de primer orden fueron especificados como indicadores de factores de segundo orden correspondientes a las tres dimensiones del perdón disposicional. Adicionalmente, se especificaron dos factores de valencia afectiva, que acumulan la varianza producto de la dirección de los ítems. Los factores que reflejan la valencia de los ítems se especificaron como no correlacionados con otros factores de segundo orden. Las cargas de cada uno de los factores de segundo orden asociadas a los factores de valencia se restringieron a ser iguales al igual que en el estudio de Thompson et al. (2005).

\section{Resultados}

En la Tabla 1 se presentan los índices de ajuste de los tres modelos evaluados.

Los índices de ajuste muestran que el modelo de tres factores evidencia un pobre ajuste a los datos $\quad(\mathrm{CFI}<.95 ; \quad$ TLI $<.95 ; \quad$ RMSEA $>.08)$. Asimismo, al comparar el Modelo B con el C, el modelo de 6 factores obtuvo un mejor ajuste que el modelo bifactor de segundo orden de Thompson et al. (2005). Dado el mejor ajuste del modelo $\mathrm{B}$, su carácter más parsimonioso $(\mathrm{gl}=128)$ y su coherencia con la distinción sustantiva entre la dimensión positiva y negativa del perdón, se optó por retener este modelo. La Figura 1 muestra el diagrama del modelo final de 6 factores.

El examen de las saturaciones obtenidas da cuenta que estas fueron significativas $y$ en magnitudes apropiadas, manteniéndose entre los valores de .781 y .503 . Adicionalmente, todas las correlaciones entre los factores son significativas, a excepción de la correlación entre perdón a sí mismo negativo y perdón hacia otros positivo.

En segundo lugar y con el propósito de proporcionar mayor evidencia que apoyase la existencia de una estructura de seis factores, los datos se sometieron a un examen de invarianza factorial a través de un análisis confirmatorio multigrupo (MCFA), considerando como variable de agrupación el sexo. La Tabla 2 presenta los resultados de los índices de ajuste de los distintos niveles de invarianza. 
Tabla 1. Índices de ajuste de los modelos alternativos

\begin{tabular}{lcccccl}
\hline & $\chi^{2}$ & $\mathrm{df}$ & $p$ & CFI & TLI & RMSEA (CI90) \\
\hline Modelo A & 693.348 & 133 & $<.001$ & .604 & .544 & $.091(.085-.098)$ \\
Modelo B & 188.465 & 120 & $<.001$ & .952 & .938 & $.034(.024-.043)$ \\
Modelo C & 218.064 & 128 & $<.001$ & .936 & .924 & $.037(.029-.046)$ \\
\hline
\end{tabular}

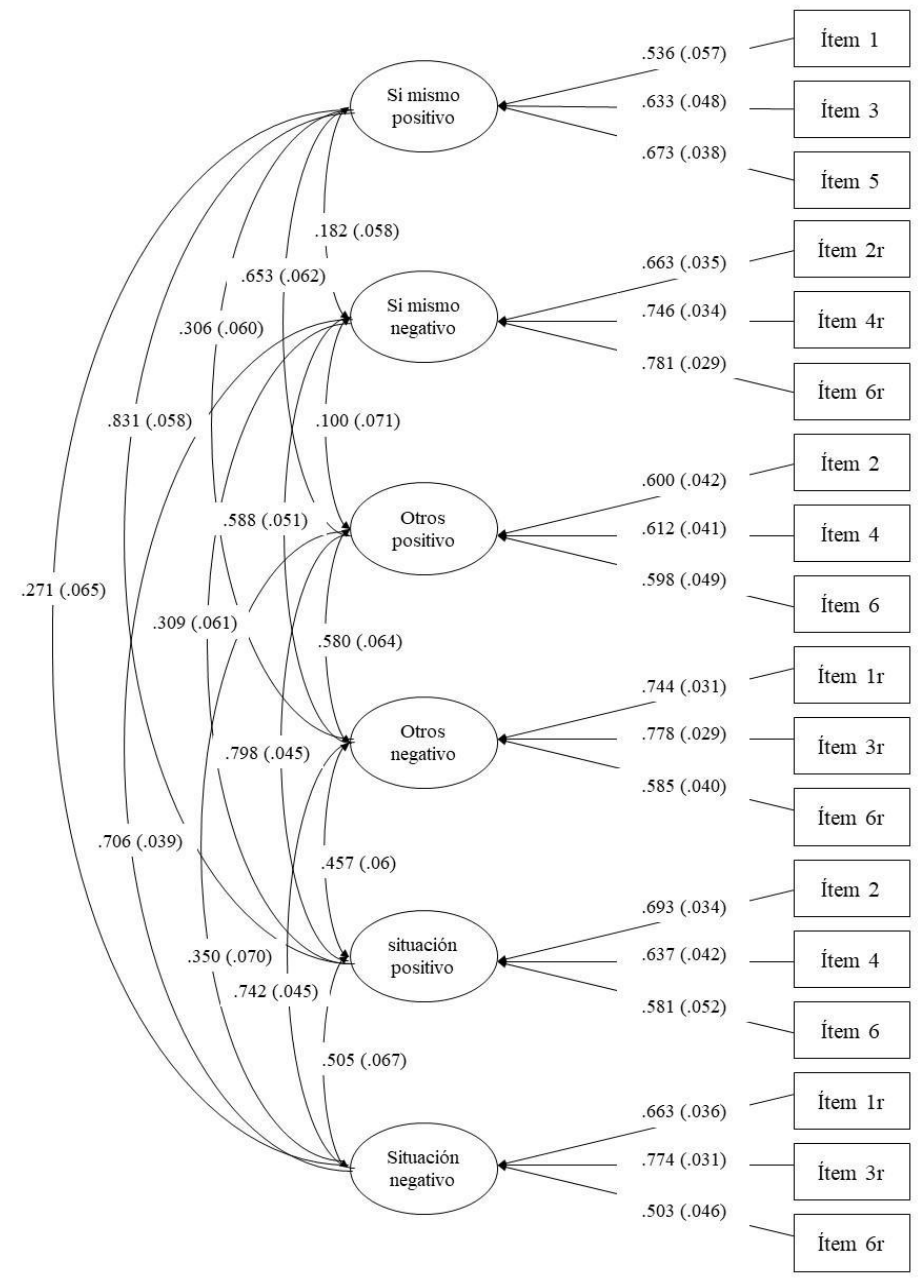

Figura 1. Solución factorial del modelo final con la solución de 6 factores relacionados. Los errores de estimación se presentan entre paréntesis

Como es posible observar, los modelos correspondientes a los tres niveles de invarianza presentan un ajuste razonable. Adicionalmente, al comparar los índices de los distintos niveles de invarianza, los resultados muestran que no existe una pérdida significativa del ajuste $\left(\Delta_{C F I}<.01\right.$; $\left.\Delta_{T L I}<.01 ; \Delta_{R M S E A}<.01 ; \Delta_{p}>.05\right)$. Por tanto, estos hallazgos soportan la existencia de equivalencia/ invarianza de medida para la escala HFS entre hombres y mujeres.

A fin de examinar si existen asociaciones diferenciales al hacer la distinción en función de la valencia positiva y negativa del perdón, se calcularon los coeficientes de correlación entre la HFS, la escala TRIM-18 y las medidas de salud mental y satisfacción con la vida. La Tabla 3 muestra las correlaciones obtenidas entre las dimensiones del HSF y TRIM-18. En términos generales, se observa que el perdón a sí mismo, a otros y a las situaciones se asocia en las direcciones esperadas a la evitación, la venganza y la benevolencia. No obstante, al distinguir según la dimensión positiva y negativa, se evidencia que la magnitud de las asociaciones difiere e incluso en algunos casos no se detectó una asociación estadísticamente significativa. Es el caso de la tendencia a la evitación del TRIM-18, que no se asoció a la dimensión positiva del perdón a sí mismo ni del perdón a las situaciones, aunque sí tuvo relación con la dimensión negativa de ambos 
Tabla 2. Análisis de Invarianza Factorial. Índices de bondad de ajuste de los modelos anidados

\begin{tabular}{llllllllll}
\hline & $\chi^{2}$ & $\mathrm{gl}$ & $p$ & CFI & TLI & RMSEA (CI95) & $\Delta \chi^{2}$ & $\Delta \mathrm{gl}$ & $\Delta p$ \\
\hline Configural & 303.520 & 240 & .003 & .947 & .933 & $.032(.019-.043)$ & & & \\
Métrica & 315.633 & 252 & .004 & .947 & .936 & $.031(.019-.042)$ & 13.969 & 12 & .303 \\
Escalar & 334.261 & 270 & .005 & .947 & .940 & $.031(.018-.041)$ & 18.748 & 18 & .408 \\
\hline
\end{tabular}

Tabla 3. Correlaciones entre el TRIM-18 y las dimensiones de la HFS

\begin{tabular}{llccc}
\hline & & \multicolumn{3}{c}{ TRIM-18 } \\
\cline { 2 - 4 } Perdón a sí mismo & Dimensión & Evitación & Venganza & Benevolencia \\
\hline & $\begin{array}{l}\text { Positiva } \\
\text { Dimensión }\end{array}$ & -.047 & $-.219^{*}$ & $.173^{*}$ \\
& Negativa & $-.106^{*}$ & $-.202^{*}$ & -.025 \\
Dimensión & $-.246^{*}$ & $-.284^{*}$ & $.379^{*}$ \\
Perdón a otros & $\begin{array}{l}\text { Positiva } \\
\text { Dimensión } \\
\text { Negativa }\end{array}$ & $-.338^{*}$ & $-.484^{*}$ & $.245^{*}$ \\
Perdón a situaciones & $\begin{array}{l}\text { Dimensión } \\
\text { Positiva } \\
\text { Dimensión } \\
\text { Negativa }\end{array}$ & -.035 & $-.236^{*}$ & $.155^{*}$ \\
\hline
\end{tabular}

Nota. ${ }^{*} p<.05$.

Tabla 4. Correlaciones entre las dimensiones de la HFS y síntomas de salud mental y satisfacción con la vida

\begin{tabular}{llcccc}
\hline & & Depresión & Ansiedad & Estrés & Satisfacción con la vida \\
\hline \multirow{2}{*}{ Perdón a sí mismo } & $\begin{array}{l}\text { Dimensión } \\
\text { Positiva }\end{array}$ & $-.117^{*}$ & -.057 & -.010 & $.200^{*}$ \\
& $\begin{array}{l}\text { Dimensión } \\
\text { Negativa }\end{array}$ & $-.375^{*}$ & $-.318^{*}$ & $-.366^{*}$ & $.261^{*}$ \\
& $\begin{array}{l}\text { Dimensión } \\
\text { Perdón a otros }\end{array}$ & -.047 & -.072 & -.050 & $.203^{*}$ \\
& $\begin{array}{l}\text { Positiva } \\
\text { Dimensión } \\
\text { Negativa }\end{array}$ & $-.201^{*}$ & $-.225^{*}$ & $-.240^{*}$ & $.234^{*}$ \\
Perdón a situaciones & $\begin{array}{l}\text { Dimensión } \\
\text { Positiva } \\
\text { Dimensjón } \\
\text { Negativa }\end{array}$ & $-.200^{*}$ & $-.168^{*}$ & $-.173^{*}$ & $.279^{*}$ \\
& & $-.351^{*}$ & $-.299^{*}$ & $-.385^{*}$ & $.241^{*}$ \\
\hline
\end{tabular}

Nota. $* p<.05$.

objetos del perdón; también se observa que la subescala de benevolencia no correlacionó con la dimensión negativa del perdón a sí mismo, pero sí con la dimensión positiva.

La Tabla 4 presenta las correlaciones entre las dimensiones de la HFS, los índices de salud mental y los niveles de satisfacción con la vida. Del mismo modo que con el TRIM-18, los resultados obtenidos van, en general, en dirección de lo esperado. Las dimensiones positivas de los tres objetos del perdón disposicional correlacionan de manera directa con los índices de salud mental, aunque en magnitudes bajas, e incluso en algunos no alcanzan a ser significativas. En cambio, las dimensiones negativas de los tres objetos del perdón disposicional tienen asociaciones significativas de mayor magnitud con síntomas de ansiedad, depresión y estrés. En el caso de la relación entre las dimensiones y objetos del perdón disposicional y la satisfacción con la vida, se identificaron asociaciones tanto con las dimensiones positivas como negativas del perdón en magnitudes similares.

\section{Discusión}

El presente estudio tuvo como propósito examinar la estructura factorial de la versión chilena de la Heartland Forgiveness Scale. Los resultados obtenidos evidencian que, a diferencia del modelo jerárquico propuesto por Thompson et 
al. (2005), una estructura de seis factores es la que mejor representa los datos. La solución factorial retenida es similar en términos teóricos a la reportada por Kaleta et al. (2016), aunque en este caso corresponde a un modelo más parsimonioso, ya que no considera estructuras de segundo o tercer orden. Desde un punto de vista sustantivo, la distinción entre la dimensión positiva y negativa para cada uno de los objetos del perdón es coherente con discusiones teóricas previas (Fincham et al., 2004; Fincham, 2015; Rye et al., 2001) y representa una caracterización más avanzada del perdón (Kaleta \& Mróz, 2018b). Por tanto, según esta conceptualización, la ausencia o reducción de respuestas negativas no sería suficiente para capturar y comprender el perdón como fenómeno complejo y sostendría la noción que las dimensiones negativas y positivas serían constructos distintos aunque correlacionados y no meramente factores de método.

En apoyo a esta propuesta, se observó que las puntuaciones de la HFS se asociaron en las direcciones esperadas con las dimensiones del TRIM-18. Sin embargo, y a diferencia de lo reportado por Thompson et al. (2005), en que solo el perdón a otros y a las situaciones se asociaron al perdón episódico, pero no el perdón a sí mismo, en este caso sí se detectaron asociaciones, de distinta magnitud y significancia, al hacer la distinción entre la dimensión positiva y negativa. Además, se identificó que en general, las tres subescalas de la HFS: perdón a otros, a sí mismo y a las situaciones, se correlacionaron con la evitación, venganza y benevolencia. No obstante, se observaron diferencias en la magnitud de dichas asociaciones según la dimensión positiva y negativa, e incluso, en algunos casos, estas relaciones no fueron significativas. Ello fundamenta entonces que al tratar estas dimensiones como constructos o factores separados permite capturar matices en los resultados que no podrían ser identificados si fuesen tratados como factores de método.

Asimismo, las subescalas de la HFS se asociaron a medidas de salud mental $y$ satisfacción con la vida en las direcciones esperadas. Esto es coherente con estudios previos que han documentado que el perdón está vinculado a una menor sintomatología asociada al estrés, ansiedad o depresión (e.g., Akhtar y
Barlow, 2018; Griffin et al., 2015; Kaleta \& Mróz, 2020; Macaskill, 2012). Es de notar, sin embargo, que se detectaron asociaciones de mayor magnitud entre el perdón a sí mismo y los síntomas de salud mental que con el perdón interpersonal, cuestión que ha sido identificada previamente (Mauger et al., 1992; Kaleta \& Mróz, 2020). Este hallazgo va en la línea de lo planteado por Worthington et al. (2007), que sostienen que la auto-recriminación, la vergüenza y la culpa que suelen acompañar el reconocerse como fuente de daño para sí u otros podría afectar el propio cuidado, aumentando la vulnerabilidad a la depresión y la ansiedad.

Asimismo, se evidenció que las dimensiones negativas del perdón estuvieron más fuertemente asociadas a los indicadores de salud mental que las dimensiones de valencia positiva, lo cual podría explicarse a partir del carácter altamente estresante que tienen las reacciones negativas asociadas a la ocurrencia de una transgresión (resentimiento, rabia, culpa, entre otras) y apoya la noción que el perdón tendría un mayor impacto sobre la salud mediante la reducción del unforgiveness, que mediante el reemplazo del resentimiento por respuestas positivas (Harris \& Thoresen, 2005). Según estos hallazgos entonces, la capacidad para reestructurar la visión de sí mismo, los otros y el mundo de un modo menos hostil podría representar un factor protector más poderoso que la sola presencia de benevolencia para la salud mental. Por tanto y a la luz de lo evidenciado en el presente estudio, investigaciones futuras podrían profundizar en la contribución diferencial que hacen las dimensiones de valencia positiva y negativa del perdón disposicional en variables de bienestar y salud mental. Lo mismo aplica al considerar el impacto sobre el bienestar en relaciones afectivas cercanas, donde la presencia de respuestas más benevolentes, y no solo la reducción de las respuestas negativas, sería particularmente relevante (Fincham, 2009).

Este estudio tiene una fortaleza adicional que es el haber examinado la invarianza factorial de la HFS entre hombres y mujeres, aspecto que, hasta donde se tiene conocimiento, no había sido evaluada previamente. Los resultados obtenidos permiten concluir que el modelo de seis factores de la HFS es psicométricamente equivalente entre mujeres y hombres. Ello implica que es posible 
realizar comparaciones en los puntajes de esta escala según sexo.

En términos prácticos, la estructura factorial de seis factores de la HFS considera un sistema de puntuación más simple para su utilización tanto por investigadores aplicados como por profesionales del área clínica y de la salud. Por otra parte y en cuanto a las implicancias de este estudio, los resultados obtenidos avalan el ya reconocido carácter multidimensional del perdón y fundamentan la relevancia de examinar si la estructura conceptual original es estable en distintos contextos. Además, la detección de diferencias en la asociación de las dimensiones del perdón con constructos relevantes para la salud mental es necesaria en tanto permite identificar focos de intervención más específicos, así como bajo qué condiciones los distintos aspectos del perdón contribuyen o no y en qué medida a la salud mental.

Pese a la potencial contribución del estudio, este posee limitaciones que deben ser consideradas. El carácter no probabilístico de la muestra y la mayor representación de personas autodefinidas como heterosexuales limitan la generalización de los resultados. Futuros estudios podrían explorar si además de la equivalencia según sexo, la HFS tiene un funcionamiento similar en personas de distintas orientaciones sexuales.

A pesar de ello, este estudio permite contar con un instrumento con adecuadas propiedades psicométricas para la evaluación del perdón disposicional, aportando evidencia del comportamiento de la HFS en el contexto chileno.

\section{Referencias}

Aalgaard, R. A., Bolen, R. M., \& Nugent, W. R. (2016). A literature review of forgiveness as a beneficial intervention to increase relationship satisfaction in couples therapy. Journal of Human Behavior in the Social Environment, 26(1), 46-55. https://doi.org/10.1080/10911359.2015.10591 66

Abad, F., Olea, J. Ponsoda, V., \& García, C. (2014). Medición en ciencias sociales y de la salud. Editorial SIntesis,
Abu-Raiya, H., \& Ayten, A. (2020) Religious involvement, interpersonal forgiveness and mental health and well-being among a multinational sample of Muslims. Journal of Happiness Studies, 21(8), 3051-3067. https://doi.org/10.1007/s10902-019-00213-8

Akhtar, S., \& Barlow, J. (2018). Forgiveness therapy for the promotion of mental wellbeing: A systematic review and metaAnalysis. Trauma, Violence \& Abuse, 19(1), 107-122. https://doi.org/10.1177/1524838016637079

Antony, M., Bieling P., Cox B., Enns, M., \& Swinson R. (1998). Psychometric properties of the 42 - item and 21 - item versions of the Depression Anxiety Stress Scales in clinical groups and a community sample. Psychological Assessment, 10(2), 176-181. https://doi.org/10.1037/1040-3590.10.2.176

Berry, J. W., Worthington, E. L., Parrott, L., O'Connor, L. E., \& Wade, N. G. (2001). Dispositional forgivingness: Development and construct validity of the Transgression Narrative Test of Forgivingness (TNTF). Personality and Social Psychology Bulletin, 27(10), 1277-1290. https://doi.org/10.1177/01461672012710004

Brown, T. (2006). Confirmatory Factor Analysis for Applied Research. The Guilford Press.

Brown, R. P. (2003). Measuring individual differences in the tendency to forgive: Construct validity and links with depression. Personality and Social Psychology Bulletin, 29(6), 759-771. https://doi.org/10.1177/014616720302900600 8

Bueschel, R. K. (2010). Development of an Implicit Measure of Dispositional Forgiveness [Tesis doctoral]. Waco, Texas, Estados Unidos: Baylor University.

Bugay, A., Demir, A., \& Delevi, R. (2012). Assessment of reliability and validity of the Turkish version of Heartland Forgiveness Scale. Psychological Reports, 111(2), 575584.

https://doi.org/10.2466/08.21.PR0.111.5.575584

Consoli, S., Rossi, A., Thompson, L. Y., Volpi, C., Mannarini, S., Castelnuovo, G., \& Molinari, E. (2020). Assessing psychometric 
properties of the Italian version of the Heartland Forgiveness Scale. Frontiers in Psychology, 11.

596501.https://doi.org/10.3389/fpsyg.2020.59 6501

Dahiya, R., \& Rangnekar, S. (2018). Forgiveness in Indian organizations: A revisit of the Heartland Forgiveness Scale. Current Psychology, 39(6), 2174-2191. https://doi.org/10.338910.1007/s12144-0189879-6

Diener, E., Emmons, R., Larsen, R. J., \& Griffin, S. (1985). The satisfaction with life scale. Journal of Personality Assessment, 49(1), 7175. https://doi.org/10.1207/s15327752jpa4901_13

Enright, R. D., \& Zell, R. L. (1989). Problems encountered when we forgive one another. Journal of Psychology and Christianity, 8, 5260.

Enright, R. D. (1996). Counseling within the forgiveness triad: On forgiving, receiving, forgiveness, and self-forgiveness. Counseling and Values, 40(2), 107-126. https://doi.org/10.1002/j.2161007X.1996.tb00844.x

Enright, R. D., \& Fitzgibbons, R. P. (2015). Forgiveness therapy: An empirical guide for resolving anger and restoring hope. Washington, D.C, Estados Unidos: American Psychological Association.

Fincham, F. D. (2009). Forgiveness: Integral to a science of close relationships? In $\mathrm{M}$. Mikulincer, \& P. Shaver (Eds.), Prosocial motives, emotions, and behavior: The better angels of our nature (pp. 347-365). Washington: APA

Fincham, F. D. (2015). Forgiveness, family relationships, and health. En L. Toussaint, E. L. Worthington, \& D. Williams (Eds.), Forgiveness and Health: Scientific Evidence and Theories Relating Forgiveness to Better Health (pp. 255-270). Dordrecht, Holanda: Springer. https://doi.org/10.1007/978-94-0179993-5_17

Fincham, F. D., \& Beach, S. R. H. (2002). Forgiveness in marriage: Implications for psychological aggression and constructive communication. Personal Relationships, 9(3), 239-251. https://doi.org/10.1111/1475-6811.00016

Fincham, F. D., Beach, S. R. H., \& Davila, J. (2004). Forgiveness and conflict resolution in marriage. Journal of Family Psychology, 18(1), 72-81.

https://doi.org/10.1037/0893-3200.18.1.72

Gall, T. L., \& Bilodeau, C. (2020). The role of forgiveness as a coping response to intimate partner stress. Journal of Spirituality in Mental Health. https://doi.org/10.1080/19349637.2020.17457 25

Gallo-Giunzioni, K., Prieto-Ursúa, M., FernándezBelinchón, C., \& Luque-Reca, O. (2021). Measuring forgiveness: Psychometric properties of the Heartland Forgiveness Scale in the Spanish population. International Journal of Environmental Research and Public Health, 18(1), 1-14.

https://doi.org/10.3390/ijerph18010045

Ghaemmaghami, P., Allemand, M., \& Martin, M. (2011). Forgiveness in younger, middle-aged and older adults: Age and gender matters. Journal of Adult Development, 18(4), 192203. https://doi.org/10.1007/s10804-011-9127-x

Gordon, K. C., \& Baucom, D. H. (1998). Understanding betrayals in marriage: A synthesized model of forgiveness. Family Process, 37(4), 425-449. https://doi.org/10.1111/j.15455300.1998.00425.x

Gordon, K. C. (2003). Forgiveness and marriage: Preliminary support for a measure based on a model of recovery from a marital betrayal. The American Journal of Family Therapy, 31(3), 179-199.

https://doi.org/10.1080/01926180301115

Gordon, K. C., Hughes, F. M., Tomcik, N. D., Dixon, L. J., \& Litzinger, S. (2009). Widening spheres of impact: The role of forgiveness in marital and family functioning. Journal of Family Psychology, 23(1), 1-13. https://doi.org/10.1037/a0014354

Griffin, B. J., Worthington, E. L., Lavelock, C. R., Wade, N. G., \& Hoyt, W. T. (2015). Forgiveness and mental health. En L. Toussaint, E. L. Worthington, y D. R. Williams (Eds.), Forgiveness and Health: Scientific Evidence and Theories Relating 
Forgiveness to Better Health (pp. 77-90). Dordrecht, Holanda: Springer. https://doi.org/10.1007/978-94-017-9993-5_6

Guzmán, M. (2010). El perdón en relaciones cercanas: Conceptualización desde una perspectiva psicológica e implicancias para la práctica clínica. Psykhe, 19(1), 19-30. https://doi.org/10.4067/S071822282010000100002

Guzmán, M., Tapia, M. J., Tejada, M., \& Valenzuela, K. (2014). Evaluación del perdón en relaciones de pareja: Propiedades psicométricas del transgression-related interpersonal motivations inventory (trim-18) en un contexto chileno. Psykhe, 23(2), 1-12. https://doi.org/10.7764/psykhe.23.2.623

Guzmán-González, M., Rivera-Ottenberger, D., Garrido, L., Contreras, P., \& Yárnoz-Yaben, S. (2019). Estructura factorial, invarianza métrica y propiedades psicométricas de la versión chilena del Cuestionario de Perdón a la ex pareja. Revista Iberoamericana de Diagnóstico y Evaluación - e Avaliação Psicológica, 52(3), 41-52. http://ojs.uc.cl/index.php/psykhe/article/view/ 21299

Hall, J., \& Fincham, F. (2005). Self-forgiveness: The stepchild of forgiveness research. Journal of Social and Clinical Psychology, 24(5), 621637. https://doi.org/10.1521/jscp.2005.24.5.621

Harris, A. H. S., \& Thoresen, C. E. (2005). Forgiveness, unforgiveness, health, and disease. En E. L. Worthington (Ed.), Handbook of Forgiveness (pp. 321-333). New York, Estados Unidos: Routledge.

Ikedo, F., Castro, L., Fraguas, S., Rego, F., \& Nunes, R. (2020). Cross-cultural adaptation and validation of the European Portuguese version of the Heartland Forgiveness Scale. Health and Quality of Life Outcomes, 18(289), 1-9. https://doi.org/10.1186/s12955-020-01531-9

Jung, M., Park, Y., Baik, S. Y., Kim, C. L., Kim, H. S., \& Lee, S. H. (2019). Self-forgiveness moderates the effects of depression on suicidality. Psychiatry Investigation, 16(2), 121-129.

https://doi.org/10.30773/pi.2018.11.12.1
Kachadourian, L. K., Fincham, F., \& Davila, J. (2004). The tendency to forgive in dating and married couples: The role of attachment and relationship satisfaction. Personal Relationships, 11(3), 373-393. https://doi.org/10.1111/j.14756811.2004.00088.x

Kaleta, K., \& Mróz, J. (2018a). Forgiveness and life satisfaction across different age groups in adults. Personality and Individual Differences, 120, 17-23. https://doi.org/10.1016/j.paid.2017.08.008

Kaleta, K., \& Mróz, J. (2018b). Personality traits and two dimensions of forgivingness. Roczniki Psychologiczne, 21(2), 147-162. https://doi.org/10.18290/rpsych.2018.21.2-3

Kaleta, K., \& Mróz, J. (2020). The relationship between basic hope and depression: Forgiveness as a Mediator. Psychiatric Quarterly, 91(3), 877-886. https://doi.org/10.1007/s11126-020-09759-w

Kaleta, K., Mróz, J., \& Guzewicz, M. (2016). Polska adaptacja Skali Przebaczenia Heartland Forgiveness Scale [Polish adaptation of the Heartland Forgiveness Scale]. Przeglad Psychologiczny, 59(4), 387402.

Lee, Y.-R., \& Enright, R. D. (2019). A metaanalysis of the association between forgiveness of others and physical health. Psychology \& Health, 34(5), 626-643. https://doi.org/10.1080/08870446.2018.15541 85

Macaskill, A. (2012). Differentiating dispositional self-forgiveness from other-forgiveness: Associations with mental health and life satisfaction. Journal of Social and Clinical Psychology, 31(1), 28-50. https://doi.org/10.1521/jscp.2012.31.1.28

Martinez Arias, M., Hernandez LLoreda, M. V., \& Hernandez LLoreda, M. J. (2006). Psicometría. Alenaza Editorial.

Mauger, P. A., Perry, J. E., Freeman, T., Grove, D. C., McBride, A. G., \& McKinney, K. E. (1992). The measurement of forgiveness: Preliminary research. Journal of Psychology and Christianity, 11(2), 170-180.

McCullough, M. E., Bellah, C. G., Kilpatrick, S. D., \& Johnson, J. L. (2001). Vengefulness: Relationships with forgiveness, rumination, 
well-being, and the Big Five. Personality and Social Psychology Bulletin, 27(5), 601-610. https://doi.org/10.1177/0146167201275008

McCullough, M. E., \& Hoyt, W. T. (2002). Transgression-related motivational dispositions: Personality substrates of forgiveness and their links to the Big Five. Personality and Social Psychology Bulletin, 28(11), 1556-1573. https://doi.org/10.1177/014616702237583

McCullough, M. E., Root, L. M., \& Cohen, A. D. (2006). Writing about the benefits of an interpersonal transgression facilitates forgiveness. Journal of Consulting and Clinical Psychology, 74(5), 887-897. https://doi.org/10.1037/0022-006X.74.5.887

McCullough, M. E., Root, L. M., Tabak, B., \& Witvliet, C. V. O. (2009). Forgiveness. En S. J. López, \& C. R. Snyder (Eds.), Handbook of Positive Psychology (2 ${ }^{\mathrm{a}}$ ed., pp. 427-435). New York, Estados Unidos: Oxford University Press.

McCullough, M. E., \& Witvliet, C. V. (2002). The psychology of forgiveness. En C. R. Snyder, \& S. J. López (Eds.), Handbook of Positive Psychology (p. 446-458). New York, Estados Unidos: Oxford University Press.

Miller, A. J., \& Worthington, E. L. (2010). Sex differences in forgiveness and mental health in recently married couples. The Journal of Positive Psychology, 5(1), 12-23. https://doi.org/10.1080/17439760903271140

Miller, A. J., Worthington, E. L., \& McDaniel, M. A. (2008). Gender and forgiveness: A metaanalytic review and research agenda. Journal of Social and Clinical Psychology, 27(8), 843876. https://doi.org/10.1521/jscp.2008.27.8.843

Muñiz, J., Elosua, P., \& Hambleton, R. K. (2013). Directrices para la traducción y adaptación de los tests: segunda edición. Psicothema, 25(2), 151-157. https://doi.org/10.7334/psicothema2013.24

Muñiz, J., \& Hambleton, R. (1996). Directrices para la traducción y adaptación de los tests. Papeles del Psicólogo, 66. 63-70.

Muthén, L. K., \& Muthén, B. O. (1998-2015). Mplus statistical modeling software: Release 7.2. Los Angeles, California, Estados Unidos: Muthén \& Muthén.
Osanai, A., \& Furukawa, M. (2005). Development of a Japanese version of the Heartland Forgiveness Scale. Annual Bulletin of Psychological Studies, Showa Women's University, 8(5), 51-57.

Rosales, R. M., Rivera, S., Reidl, L. M., \& García, M. (2018). Perdón y Resentimiento hacia la Pareja: Desarrollo y Validación de Dos Escalas. Revista Iberoamericana de Diagnóstico y Evaluación - e Avaliação Psicológica, 2(47), 141-156. https://doi.org/10.21865/RIDEP47.2.10

Rye, M. S., Loiacono, D. M., Folck, C. D., Olszewski, B. T., Heim, T. A., \& Madia, B. P. (2001). Evaluation of the psychometric properties of two forgiveness scales. Current Psychology, 20(3), 260-277. https://doi.org/10.1007/s12144-001-1011-6

Rey, L., \& Extremera, N. (2016). Agreeableness and interpersonal forgiveness in young adults: The moderating role of gender. Terapia Psicologica, 34(2), 103-110. http://dx.doi.org/10.4067/S071848082016000200003

Sheldon, P., Gilchrist-Petty, E., \& Adam, J. (2014). You did what? The relationship between forgiveness tendency, communication of forgiveness, and relationship satisfaction in married and dating couples. Communication Reports, 27(2), 7890.

https://doi.org/10.1080/08934215.2014.902486

Sireci, S. G. (1998). Gathering and analyzing content validity data. Educational Assessment, 5(4), 299-321. doi:10.1207/s15326977ea0504_2

Sireci, S. G. (2003). Validity content. En Ballesteros, R. F. (Ed.). Encyclopedia of psychological assessment. SAGE.

Strelan, P., \& Covic, T. (2006). A review of forgiveness process models and a coping framework to guide future research. Journal of Social and Clinical Psychology, 25(10), 1059-1085.

https://doi.org/10.1521/jscp.2006.25.10.1059

Suen, H. K. (1990). Principles of test theories. Hillsdale, NJ. Lawrence Erlbaum.

Thompson, L. Y., \& Snyder, C.R. (2003). Measuring forgiveness. En Lopez, J.S. \& Snyder, C. R. (Eds.). Positive Psychological 
Assessment. A Handbook of Models and Measures. Washington, D.C, Estados Unidos: American Psychological Association. https://doi.org/10.1037/10612-000

Thompson, L. Y., Snyder, C. R., Hoffman, L., Michael, S. T., Rasmussen, H. N., Billings, L. S., \& Roberts, D. E. (2005). Dispositional forgiveness of self, others, and situations. Journal of Personality, 73(2), 313-360. https://doi.org/10.1111/j.14676494.2005.00311.x

Tuck, I., \& Anderson, L. (2014). Forgiveness, flourishing and resilience: The influences of expressions of spirituality on mental health recovery. Issues in Mental Health Nursing, 35(4), 277-282. https://doi.org/10.3109/01612840.2014.88562 3

Vera-Villarroel, P., Urzúa, A., Pavez, P., CelisAtenas, K., \& Silva, J. (2012). Evaluation of subjective well-being: Analysis of the Satisfaction with Life Scale in Chilean population. Universitas Psychologica, 11(3), 719-727.

https://doi.org/10.11144/Javeriana.upsy113.eswa

Vinet, E., Rehbein, L., Román F., \& Saiz, J. (2012). Escalas de depresión, ansiedad y estrés (DASS - 21): Validación de la versión abreviada en estudiantes universitarios chilenos. Terapia Psicológica, 30(3), 49-55. https://doi.org/10.4067/S071848082012000300005

Wade, N. G., Hoyt, W. T., Kidwell, J. E. M., \& Worthington, E. L. (2014). Efficacy of psychotherapeutic interventions to promote forgiveness: A meta-analysis. Journal of Consulting and Clinical Psychology, 82(1), 154-170. https://doi.org/10.1037/a0035268

Wade, N. G., \& Worthington, E. L. (2003). Overcoming interpersonal offenses: Is forgiveness the only way to deal with unforgiveness? Journal of Counseling and Development, 81(3), 343-353. https://doi.org/10.1002/j.15566678.2003.tb00261.x

Wilkowski, B. M., Robinson, M. D., \& TroopGordon, W. (2010). How does cognitive control reduce anger and aggression? The role of conflict monitoring and forgiveness processes. Journal of Personality and Social Psychology, 98(5), 830-840, https://doi.org/10.1037/a0018962

Worthington, E. L., \& Sandage, S. J. (2016). Forgiveness and spirituality in psychotherapy: A relational approach. Washington, D.C: American Psychological Association.

Worthington, E. L., \& Wade, N. G. (1999). The psychology of unforgiveness and forgiveness and implications for clinical practice. Journal of Social and Clinical Psychology, 18(4), 385-418. https://doi.org/10.1521/jscp.1999.18.4.385

Worthington, E. L., Witvliet, C. V. O., Pietrini, P., \& Miller, A. J. (2007). Forgiveness, health, and well-being: A review of evidence for emotional versus decisional forgiveness, dispositional forgivingness, and reduced unforgiveness. Journal of Behavioral Medicine, 30(4), 291-302. https://doi.org/10.1007/s10865-007-9105-8 